\title{
Chiral Extrapolation of the nucleon-nucleon S-wave scattering lengths
}

\author{
Jaume Tarrús Castellà* \\ Departament d'Estructura i Constituents de la Matèria and Institut de Ciències del Cosmos \\ E-mail: tarrus@ecm.ub.edu
}

\begin{abstract}
We report on a calculation of the pion mass dependence of the inverse scattering length of the nucleon-nucleon system, in the framework of a Chiral effective theory with dibaryon fields. We show that in ${ }^{3} S_{1}$ channel certain sets of diagrams with one radiation pion and an arbitrary number of potential pions are of order $\mathscr{O}(1)$, but their contributions cancel out. We discuss the difficulties of extending the proof to the ${ }^{1} S_{0}$ channel. We apply our results to chiral extrapolations of current lattice data.
\end{abstract}

The 7th International Workshop on Chiral Dynamics,

August 6 -10, 2012

Jefferson Lab, Newport News, Virginia, USA

${ }^{*}$ Speaker. 


\section{Introduction}

The size of the nucleon-nucleon S-wave scattering lengths is larger than expected from standard arguments of chiral counting, and understanding their values from QCD is still a major challenge [1]. Lattice calculations at physical light quark masses are very costly (although they have recently been carried out for some observables) and, hence, the use of chiral extrapolations will be needed for some time in order to obtain reliable estimates [2,3]. The quark mass dependence of low-energy observables can be obtained from suitable chiral effective theories.

The introduction of dibaryon fields in nucleon-nucleon effective field theories (NNEFT) is a very convenient way of implementing large scattering lengths [5-7]. It was already noticed in [9] that beyond next-to-leading order (NLO), part of the calculation must be organized in powers of $\sqrt{m_{\pi} / \Lambda_{\chi}}, m_{\pi}$ being the pion mass and $\Lambda_{\chi}$ a typical hadronic scale, rather than in powers of $m_{\pi} / \Lambda_{\chi}$ [7]. It is in fact an accident due to Wigner symmetry that the would-be $\mathscr{O}\left(m_{\pi}^{3 / 2} / \Lambda_{\chi}^{3 / 2}\right)$ correction vanishes [7, 10]. In addition, it was pointed out in Ref. [11] that the terms giving corrections $\sqrt{m_{\pi} / \Lambda_{\chi}}$ were generically large. In this paper we show that these terms can be summed up in the ${ }^{3} S_{1}$ channel and, furthermore, that they give a vanishing contribution to the scattering length. This allows us to provide a reliable chiral extrapolation formula for the inverse scattering length including terms up to order $m_{q}^{3 / 2} / \Lambda_{\chi}^{1 / 2}, m_{q}$ being the average light quark masses. Unfortunately neither the arguments that allow the resummation nor the proof that the effect vanishes apply to the ${ }^{1} S_{0}$ channel.

\section{NNEFT with Dibaryon fields}

Our starting point is the effective field theory (EFT) for the the baryonic number two sector of QCD for energies much smaller than $\Lambda_{\chi}$, proposed in Ref. [6, 7]. The distinct feature of this EFT is that, in addition to the usual degrees of freedom for a NNEFT theory, namely nucleons and pions, two dibaryon fields, an isovector $\left(D_{s}^{a}\right)$ with quantum numbers ${ }^{1} S_{0}$ and an isoscalar $\left(\vec{D}_{v}\right)$ with quantum numbers ${ }^{3} S_{1}$, are also included. Chiral symmetry, and its breaking due to the quark masses in QCD, constrain the possible interactions of the nucleons and dibaryon fields with the pions. When we restrict ourselves to energies $E \gtrsim m_{\pi}$, we can integrate out nucleons and pions of such energies. The only pionic degrees of freedom remaining are the so-called potential pions, that is pions with $q^{0} \sim \mathbf{q}^{2} / m_{N}$, where $m_{N}$ is the nucleon mass. We call the EFT for this energy region potential NNEFT (pNNEFT). For $p \lesssim m_{\pi}^{2} / \Lambda_{\chi}$ we can integrate out the potential pions (as well as the nucleons with such momenta). Thus the EFT for this energy region does not have dynamical pions and we will refer to it as pionless NNEFT ( $\not$ NNEFT). In $\not$ NNEFT the expression for the scattering lengths is very simple

$$
a_{i}^{-1}=\frac{\pi \delta_{m_{i}}}{m_{N} A_{i}^{2}}, \quad i=s\left({ }^{1} S_{0}\right), v\left({ }^{3} S_{1}\right),
$$

where $\delta_{m_{i}}$ is the dibaryon residual mass, and $A_{i}$ is the low-energy constant associated to the LO nucleon-dibaryon vertex. These two low-energy constants receive matching contributions when going from the high energy NNEFT to $\not$ NNEFT. In the next section we study in detail the contributions to the residual mass with one radiation pion, the remaining matching contributions are discussed in $[7,8]$. 


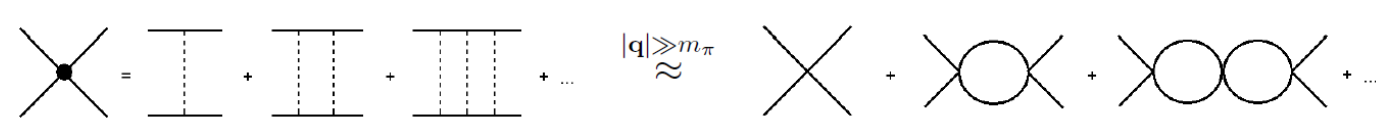

Figure 1: Potential pion exchanges in the ${ }^{1} S_{0}$ channel can be approximated by contact interactions and resummed into an effective vertex when the external momentum is bigger than the pion mass.

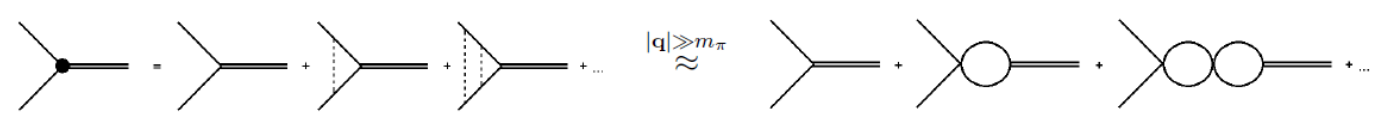

Figure 2: Resummation of potential pions in the dibaryon-nucleon vertex.

\section{Potential pions in loops with radiation pions}

We discuss in this section this particular class of contributions to the residual mass of the matching calculation of NNEFT with pNNEFT involving radiation pions. The lowest order diagrams involving one radiation pions cancel due to Wigner symmetry. When a potential pion, in this case a pion with $q^{0} \sim m_{\pi}$ and $\mathbf{q} \sim \sqrt{m_{\pi} m_{N}}$, is added to one of those diagrams a parametric suppression of only $\sqrt{m_{\pi} / \Lambda_{\chi}}$ occurs [7,10], which numerically turns out to be $\mathrm{O}(1)[8,11]$. It is then necessary to sum up these kinds of contributions.

\subsection{Loop resummation}

Let us consider the exchange of $n$ potential pions between two nucleon lines. If we project it to the ${ }^{1} S_{0}$ channel, the three-momenta coming from the vertices of each potential pion exchange contract between themselves. Note that this is not the case if we project to the ${ }^{3} S_{1}$ channel, where a three-momentum from one of the vertices of a given potential pion exchange may get contracted with a three-momentum of a neighboring potential pion exchange vertex. If these $n$-pion exchanges are in a loop with a radiation pion, then the three-momentum in the denominator of the potential pion propagators dominates over the pion mass and the pion energy. As a consequence, the potential pion exchanges collapse into a local vertices. Again, this is not so in the ${ }^{3} S_{1}$ channel, where even at very large momentum transfer the potential remains non-local. In the left hand side of Fig. 1 we depicted the first terms in a series of diagrams with an arbitrary large number of potential pion exchanges. Using the previous reasoning we can collapse the potential pion exchanges into local vertices obtaining the diagrams on the right hand side. Naively we would expect each bubble to suppress the diagram by a factor of $\sqrt{m_{\pi} / \Lambda_{\chi}}$. However a more careful analysis shows that the actual size of each bubble is in fact $\sqrt{m_{\pi}} / \alpha \sim 1.19$, and hence the series should be resummed. The result of the resummation can be cast as an effective energy-dependent four-nucleon vertex with coupling constant

$$
C_{e f f}=i \frac{g_{A}^{2}}{2 f_{\pi}^{2}} \frac{\alpha}{\alpha+\sqrt{q^{0}-i \varepsilon}},
$$

where we have taken the external energy to be $-q^{0}$, and $\alpha=8 \pi f_{\pi}^{2} /\left(g_{A}^{2} m_{N}^{3 / 2}\right)$. An analogous resummation has to be done for potential pion exchanges in the nucleon-dibaryon vertex of Fig. 2. Furthermore, using the effective vertex of Eq. (3.1) we can construct the self-energy depicted in Fig. 3, which inside radiation pion loops turns out to be of order $\mathscr{O}(1)$ and thus has to be included in the LO propagator. Note that in order to have a ${ }^{1} S_{0}$ nucleon-nucleon state in a loop with a single radiation pion, the initial nucleon-nucleon state must be in the ${ }^{3} S_{1}$ channel. 


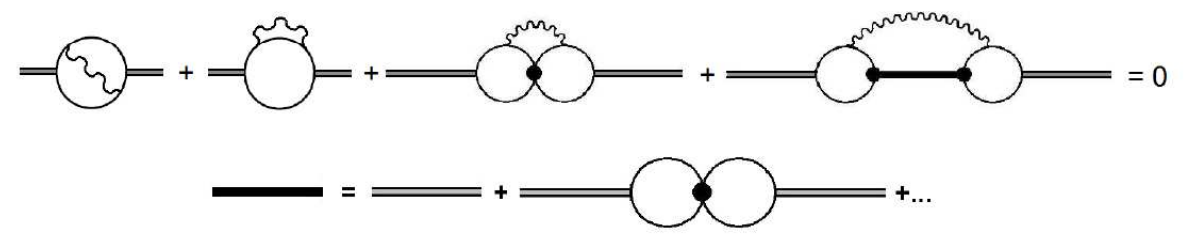

Figure 3: Order $\mathscr{O}\left(m_{\pi}^{2} / \Lambda_{\chi}\right)$ contributions to the dibaryon residual mass. Inside radiation pion loops the ${ }^{1} S_{0}$ receives an additional self-energy contribution.

\subsection{Cancellation of the contributions to $a^{3} S_{1}$}

Making use of the new effective vertices we can summarize all the contributions with one radiation pion and any number of potential pions in the diagrams of Fig. 3. The first two diagrams are given by

$$
\mathscr{A}_{1+2}=-8 A_{v}^{2} \frac{1}{\alpha} B(5 / 4,0)
$$

and the third and fourth ones

$$
\begin{aligned}
& \mathscr{A}_{3}=8 A_{v}^{2}\left(\frac{1}{\alpha} B(1 / 4,1)-\alpha^{2} B(1,1)+\alpha B(3 / 4,1)-B(1 / 2,1)\right) \\
& \mathscr{A}_{4}=8 A_{v}^{2}\left(B(1 / 2,1)-\alpha B(3 / 4,1)+\alpha^{2} B(0,2)-\alpha^{3} B(1 / 4,2)-\alpha^{6} B(1,2)+\alpha^{7} B(5 / 4,2)\right),
\end{aligned}
$$

with $B\left(\beta_{1}, \beta_{2}\right)$ defined as

$$
B\left(\beta_{1}, \beta_{2}\right)=\left(\mu^{2}\right)^{d-4} \int \frac{d^{d-1} q}{(4 \pi)^{d-1}} \frac{\mathbf{q}^{2}}{\left(\mathbf{q}^{2}+m_{\pi}^{2}\right)^{\beta_{1}}} \frac{1}{\left(\mathbf{q}^{2}+m_{\pi}^{2}-\alpha^{4}\right)^{\beta_{2}}} .
$$

These contributions are of $\mathscr{O}\left(m_{\pi}^{2} / \Lambda_{\chi}\right)$, the same order as the bare residual masses. The sum of these three contributions $\left(\mathscr{A}_{1+2}, \mathscr{A}_{3}, \mathscr{A}_{4}\right)$ adds up to zero, which can be checked by making use of the relation

$$
B\left(\beta_{1}-1, \beta_{2}\right)=B\left(\beta_{1}, \beta_{2}-1\right)+\alpha^{4} B\left(\beta_{1}, \beta_{2}\right) .
$$

The reason for this cancellation is that the contact four-nucleon interaction can be removed by the following local field redefinition of the dibaryon field: $D_{s}^{a} \rightarrow D_{s}^{a}-g_{A}^{2} /\left(2 f_{\pi}^{2} A_{s}\right) N^{T} P_{a}^{1} S_{0} N$ where $P_{a}^{1} S_{0}$, is the projector to the ${ }^{1} S_{0}$ partial wave. As we have mentioned in the previous section, the resummation cannot be carried out for the analogous diagrams for $a^{1} S_{0}$. However, it is likely that the perturbative expansion also breaks down in this channel due to numerical factors coming from loop integrals. Hence, any prediction for the quark mass dependence of $a^{1} S_{0}$ in terms of a perturbative expansion has to be taken with caution, because it could be missing large corrections. Part of the reasoning we have used in the ${ }^{3} S_{1}$ channel can be adapted to discuss the result for the diagrams with a single potential pion exchange in a loop with a radiation pion in the ${ }^{1} S_{0}$ channel. In this set of diagrams we can approximate the potential exchange by a four-nucleon contact term, and following the same reasoning as for the contributions to $a^{3} S_{1}$. The contact term can then be eliminated by a field redefinition for the $\vec{D}_{v}$ dibaryon field. We then conclude that the sum of the diagrams must vanish. 


\begin{tabular}{|c|c|c|c|}
\hline \hline & $\zeta_{1}$ & $\zeta_{2}$ & $\zeta_{3}$ \\
\hline${ }^{1} S_{0}$ & $\frac{\pi \delta_{m_{s}}^{\prime}}{m_{N} A_{s}^{2}}$ & $\frac{2 \pi\left(\left(s_{1}+s_{2}\right) / B_{0}-8 c_{1}\right)}{m_{N} A_{s}^{2}}$ & $\frac{g_{A}^{2}}{16 m_{N} f_{\pi}^{2}}\left(\frac{1}{A_{v}^{2}}+\frac{2 c_{s v}}{g_{A} A_{s} A_{v}}-\frac{3}{A_{s}^{2}}\right)-\frac{g_{A}^{2}}{4 f_{\pi}^{2}} \frac{\left(s_{1}+s_{2}\right) / B_{0}-8 c_{1}}{A_{s}^{2}}+\left(\frac{g_{A}^{2} m_{N}}{f_{\pi}^{2}}\right)^{2} \frac{\log (2)}{128 \pi^{2}}$ \\
${ }^{3} S_{1}$ & $\frac{\pi \delta_{m_{v}}^{\prime}}{m_{N} A_{v}^{2}}$ & $\frac{2 \pi\left(v_{1} / B_{0}-8 c_{1}\right)}{m_{N} A_{s}^{2}}$ & $\frac{g_{A}^{2}}{16 m_{N} f_{\pi}^{2}}\left(\frac{1}{A_{s}^{2}}+\frac{2 c_{s v}}{g_{A} A_{s} A_{v}}-\frac{3}{A_{v}^{2}}\right)-\frac{g_{A}^{2}}{4 f_{\pi}^{2}} \frac{\left(v_{1} / B_{0}-8 c_{1}\right)}{A_{v}^{2}}+5\left(\frac{g_{A}^{2} m_{N}}{f_{\pi}^{2}}\right)^{2} \frac{6+13 \log (2)}{256 \pi^{2}}$ \\
\hline \hline
\end{tabular}

Table 1: Independent free parameters in terms of the effective theory low energy constants.
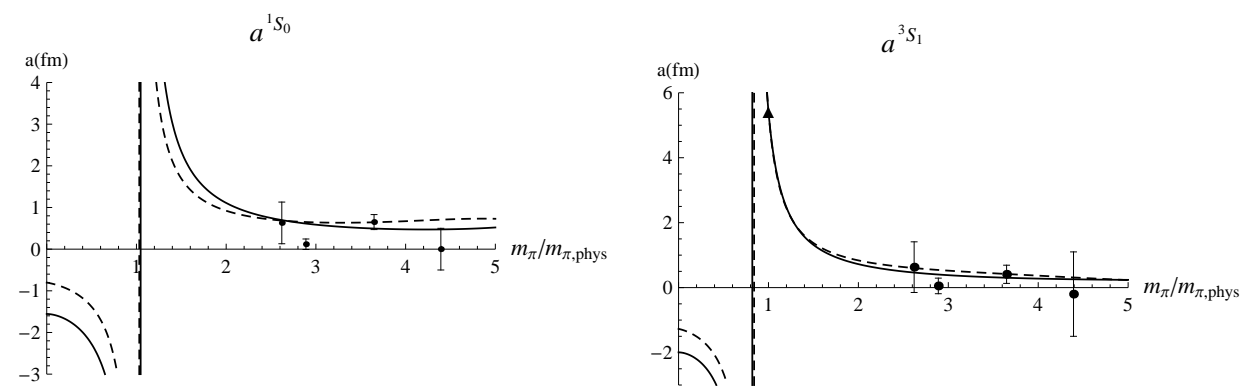

Figure 4: Plots of $a^{1} S_{0}$ (left) and $a^{3} S_{1}$ (right). The solid and dashed lines correspond to the LO and NLO respectively. The triangular dot in the $a^{3} S_{1}$ figure corresponds the physical value of the scattering length.

\begin{tabular}{|c|c|c|c|c|c|c|c|c|}
\hline \hline $\mathrm{LO}$ & $\chi_{\text {d.o.f }}^{2}$ & $\zeta_{1}(\mathrm{MeV})$ & $\zeta_{2}\left(\mathrm{MeV}^{-1}\right)$ & $\mathrm{NLO}$ & $\chi_{\text {d.o.f }}^{2}$ & $\zeta_{1}(\mathrm{MeV})$ & $\zeta_{2}\left(\mathrm{MeV}^{-1}\right)$ & $\zeta_{3}\left(\mathrm{MeV}^{-2}\right)$ \\
\hline${ }^{1} S_{0}$ & 3.74 & -126 & $0.67 \cdot 10^{-3}$ & ${ }^{1} S_{0}$ & 2.4 & -246 & $4.56 \cdot 10^{-3}$ & $9.21 \cdot 10^{-6}$ \\
${ }^{3} S_{1}$ & 0.91 & -98 & $1.59 \cdot 10^{-3}$ & ${ }^{3} S_{1}$ & 0.4 & -155 & $3.83 \cdot 10^{-3}$ & $10.1 \cdot 10^{-6}$ \\
\hline \hline
\end{tabular}

Table 2: Parameters results from the fit.

\section{Comparison with lattice data}

The expressions for the scattering lengths can be rewritten to collect all the parameters into three independent ones [8],

$$
a_{i}^{-1}=\zeta_{i 1}\left(1-\frac{g_{A}^{2} m_{N}}{8 \pi f_{\pi}^{2}} m_{\pi}\right)+\left[\zeta_{i 2}-\frac{g_{A}^{2} m_{N}}{16 \pi f_{\pi}^{2}} \ln \left(\frac{m_{\pi}^{2}}{\mu^{2}}\right)\right] m_{\pi}^{2}+\zeta_{i 3} m_{\pi}^{3}+\frac{1}{2}\left(\frac{g_{A}^{2} m_{N}}{8 \pi f_{\pi}^{2}}\right)^{2} m_{\pi}^{3} \ln \left(\frac{m_{\pi}^{2}}{\mu^{2}}\right),
$$

$i=s\left({ }^{1} S_{0}\right), v\left({ }^{3} S_{1}\right)$. The expression obtained is quite simple and emphasizes the $m_{\pi}$ dependence. The relation of the $\zeta$ parameters to the low energy constants of the EFT can be found in Table 1. We fitted the lattice data of the NPLQCD Collaboration $[12,13]$. We forced the expressions for the scattering lengths to reproduce the experimental values at the physical pion mass, $a^{1} S_{0}=-23.7 \mathrm{fm}$ and $a^{3} S_{1}=5.38 \mathrm{fm}$. The remaining parameters have been obtained by minimizing an augmented chi-square distribution for each scattering length. The values obtained for the parameters and the chi-squared per degree of freedom are collected in Tables 2. The values obtained for $\zeta_{s 1}$ and $\zeta_{i 3}$, $i=s, v$, at NLO are on the limit of what we would consider natural size. This could indicate that significant cancellations occur at the physical pion mass in order to produce the observed values of the scattering lengths. Note that the fine tuning increases with the precision of the expression used. In the ${ }^{1} S_{0}$ channel our results in the chiral limit indicate that the scattering length remains negative, thus the system is unbounded. In the ${ }^{3} S_{1}$ channel our extrapolation of the scattering length to the chiral limit shows that it evolves from positive values at the physical pion mass to negative values, hence going from a bounded nucleon-nucleon system to an unbounded one. 


\section{Conclusions}

We have showed that contributions to the dibaryon residual masses involving an arbitrary number of potential pion exchanges in loops with radiation pions can be computed in the ${ }^{3} S_{1}$ channel. These are all of $\mathscr{O}(1)$ and have to be resummed, the result of the resummation takes the form of a contact interaction. We showed that by performing dibaryon local field redefinitions we can get rid of the contact interaction, and hence the contribution of all diagrams involving these potential pion exchanges must be zero. In the ${ }^{1} S_{0}$ channel it has not been possible for us to compute the contribution of an arbitrary number of potential pions in a loop with a radiation pion. However, similar arguments still apply to the diagrams with only one potential pion, which should then add up to zero. It is very likely that in the ${ }^{1} S_{0}$ channel the perturbative series breaks down as in the ${ }^{3} S_{1}$ channel, which means that it is possible that we are missing large contributions. We have given chiral extrapolation formulas for $1 / a^{1} S_{0}$ and $1 / a^{3} S_{1}$ up to corrections of order $\mathscr{O}\left(m_{\pi}^{3} / \Lambda_{\chi}^{2}\right)$. In section 4 we carried out a fit of these expressions to lattice data. The results show that our expressions for $a^{3} S_{1}$ are much more compatible with lattice data than those for $a^{1} S_{0}$, which could indicate that the missing, potentially large, contributions to $a^{1} S_{0}$ previously mentioned do exist. Using these results to extrapolate the scattering lengths in the chiral limit, we obtain that $a^{1} S_{0}$ keeps its negative sign, while $a^{3} S_{1}$ changes from positive to negative.

\section{Acknowledgments}

We have been supported by the CPAN CSD2007-00042 Consolider-Ingenio 2010 program (Spain), the 2009SGR502 CUR grant (Catalonia) and the FPA2010-16963 project (Spain). JT acknowledges a MEC FPU grant (Spain).

\section{References}

[1] S. R. Beane et al. [NPLQCD Collaboration], arXiv:1109.2889 [hep-lat].

[2] E. Epelbaum, U. G. Meissner and W. Gloeckle, Nucl. Phys. A 714, 535 (2003) [arXiv:nucl-th/0207089].

[3] S. R. Beane and M. J. Savage, Nucl. Phys. A 717, 91 (2003) [arXiv:nucl-th/0208021].

[4] S. R. Beane et al. Nucl. Phys. A 700, 377 (2002) [arXiv:nucl-th/0104030].

[5] S. R. Beane and M. J. Savage, Nucl. Phys. A 694, 511 (2001) [arXiv:nucl-th/0011067].

[6] J. Soto and J. Tarrus, Phys. Rev. C 78, 024003 (2008) [arXiv:0712.3404 [nucl-th]].

[7] J. Soto and J. Tarrus, Phys. Rev. C 81, 014005 (2010) [arXiv:0906.1194 [nucl-th]].

[8] J. Soto and J. Tarrus, Phys. Rev. C 85, 044001 (2012) [arXiv:1112.4426 [nucl-th]].

[9] S. Fleming, T. Mehen and I. W. Stewart, Nucl. Phys. A 677, 313 (2000) [arXiv:nucl-th/9911001].

[10] T. Mehen, I. W. Stewart and M. B. Wise, Phys. Rev. Lett. 83, 931 (1999) [arXiv:hep-ph/9902370].

[11] J. Mondejar and J. Soto, Eur. Phys. J. A 32, 77 (2007) [arXiv:nucl-th/0612051].

[12] S. R. Beane et al. Phys. Rev. Lett. 97, 012001 (2006) [arXiv:hep-lat/0602010].

[13] S. R. Beane et al. [NPLQCD Collaboration], Phys. Rev. D 81, 054505 (2010) [arXiv:0912.4243 [hep-lat]]. 